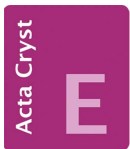

CRYSTALLOGRAPHIC COMMUNICATIONS

ISSN 2056-9890

Received 20 January 2020

Accepted 10 February 2020

Edited by M. Weil, Vienna University of Technology, Austria

Keywords: crystal structure; phosphate; $A M M^{\prime}\left(\mathrm{PO}_{4}\right)_{2}$ family; trigonal-bipyramidal coordination; isotypism.

CCDC reference: 1983244

Supporting information: this article has supporting information at journals.iucr.org/e
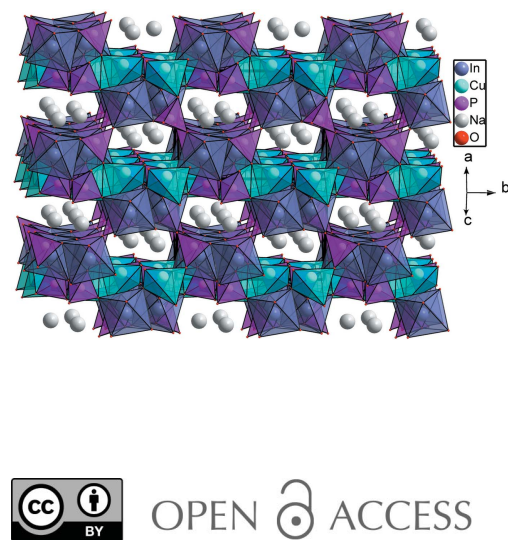

\section{Synthesis and crystal structure of $\mathrm{NaCuIn}\left(\mathrm{PO}_{4}\right)_{2}$}

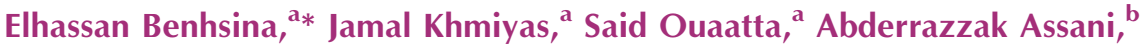 \\ Mohamed Saadib and Lahcen El Ammari ${ }^{b}$
}

\begin{abstract}
aLaboratoire de Chimie Appliquée des Matériaux, Centre des Sciences des Matériaux, Faculty of Sciences, Mohammed V University in Rabat, Avenue Ibn Batouta, BP 1014, Rabat, Morocco, and ' ${ }^{\mathbf{b}}$ Laboratoire de Chimie Appliquée des Matériaux, Centre des Sciences des Matériaux, Faculty of Sciences, Mohammed V University in Rabat, Avenue Ibn Batouta, B.P. 1014, Rabat, Morocco. *Correspondence e-mail: el_benhsina@yahoo.fr
\end{abstract}

Single crystals of sodium copper(II) indium bis[phosphate(V)], $\mathrm{NaCuIn}\left(\mathrm{PO}_{4}\right)_{2}$, were grown from the melt under atmospheric conditions. The title phosphate crystallizes in the space group $P 2_{1} / n$ and is isotypic with $\mathrm{KCuFe}\left(\mathrm{PO}_{4}\right)_{2}$. In the crystal, two $\left[\mathrm{CuO}_{5}\right]$ trigonal bipyramids share an edge to form a dimer $\left[\mathrm{Cu}_{2} \mathrm{O}_{8}\right]$ that is connected to two $\mathrm{PO}_{4}$ tetrahedra. The obtained $\left[\mathrm{Cu}_{2} \mathrm{P}_{2} \mathrm{O}_{12}\right]$ units are interconnected through vertices to form sheets that are sandwiched between undulating layers resulting from the junction of $\mathrm{PO}_{4}$ tetrahedra and $\left[\mathrm{InO}_{6}\right]$ octahedra. The two types of layers are alternately stacked along [101] and are joined into a three-dimensional framework through vertex- and edge-sharing, leaving channels parallel to the stacking direction. The channels host the sodium cations that are surrounded by four oxygen atoms in form of a distorted disphenoid.

\section{Chemical context}

Transition-metal phosphates have been the subject of intensive research as a result of their interesting physical properties and potential applications in wide-ranging fields such as catalysis, electrochemistry, luminescence (Tie et al., 1995; Pan et al., 2006; Yang et al., 2016) and ion exchange (Cheetham et al., 1999; Han et al., 2015; Manos et al., 2005, 2007; Plabst et al., 2009; Stadie et al., 2017). In these materials, the anionic framework is built up from $\mathrm{PO}_{4}$ tetrahedra linked to different kinds of transition metal $(T M)$ coordination polyhedra of the form $\left[T M \mathrm{O}_{n}\right](n=4,5$ and 6), leading to a large variety of crystal structure families. This structural diversity is mainly associated with the ability of $T M$ cations to adopt different oxidation states in various coordination polyhedra. Based on previous hydrothermal investigations aimed at orthophosphates of general formula $\left(M, M^{\prime \prime}\right)_{3}\left(\mathrm{PO}_{4}\right)_{2} \cdot n \mathrm{H}_{2} \mathrm{O}\left(M\right.$ and $M^{\prime \prime}$ = bivalent cations), we have reported on synthesis and characterization of the phosphates $\mathrm{Ni}_{2} \mathrm{Sr}\left(\mathrm{PO}_{4}\right)_{2} \cdot 2 \mathrm{H}_{2} \mathrm{O}$ (Assani et al., 2010), $\mathrm{Mg}_{1.65} \mathrm{Cu}_{1.35}\left(\mathrm{PO}_{4}\right)_{2} \cdot \mathrm{H}_{2} \mathrm{O}(\mathrm{Khmiyas}$ et al., 2015) and $\mathrm{Mn}_{2} \mathrm{Zn}\left(\mathrm{PO}_{4}\right)_{2} \cdot \mathrm{H}_{2} \mathrm{O}$ (Alhakmi et al., 2015). In this context, the aim of the present study was to develop new phases belonging to the series $A M^{\prime \prime} M^{\prime \prime \prime}\left(\mathrm{PO}_{4}\right)_{2}$ where $A, M^{\prime \prime}$ and $M^{\prime \prime \prime}$ are mono-, bi- and trivalent cations, respectively. As a result, we report here on synthesis and crystal structure of the new compound $\mathrm{NaCuIn}\left(\mathrm{PO}_{4}\right)_{2}$.

\section{Structural commentary}

The principal building units of the crystal structure of $\mathrm{NaCuIn}\left(\mathrm{PO}_{4}\right)_{2}$ are two $\mathrm{PO}_{4}$ tetrahedra linked to a $\left[\mathrm{CuO}_{5}\right]$ 


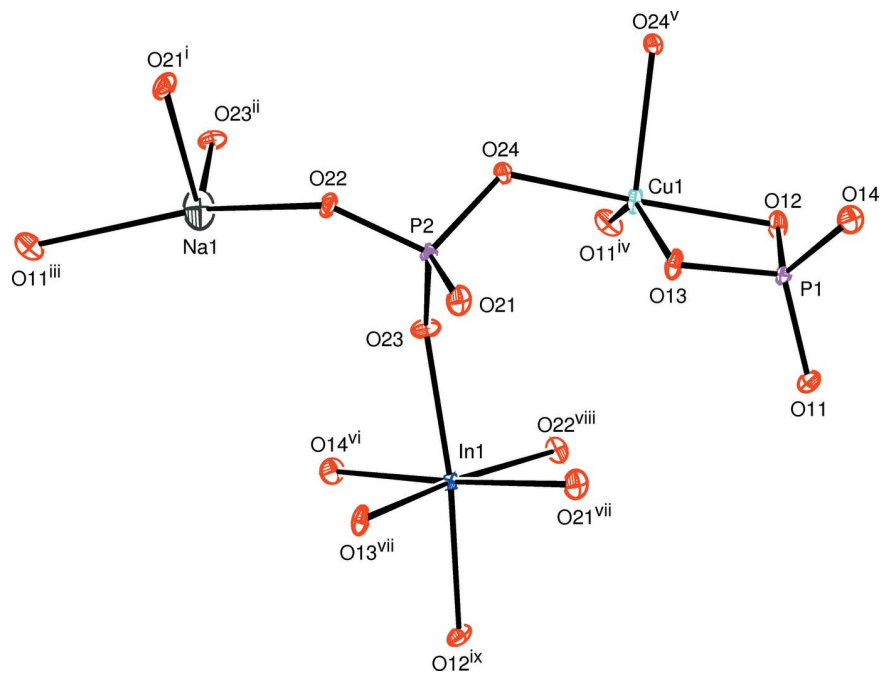

Figure 1

The principal building units in the crystal structure of $\mathrm{NaCuIn}\left(\mathrm{PO}_{4}\right)_{2}$. Displacement ellipsoids are drawn at the $50 \%$ probability level. [Symmetry codes: (i) $x+\frac{1}{2},-y-\frac{1}{2}, z+\frac{1}{2}$; (ii) $-x+1,-y,-z+2$; (iii) $-x+\frac{1}{2}, y-\frac{1}{2},-z+\frac{3}{2}$; (iv) $x+\frac{1}{2},-y+\frac{1}{2}, z+\frac{1}{2}$; (v) $-x+1,-y,-z+1$; (vi) $x$, $y, z+1$; (vii) $-x,-y,-z+1$; (viii) $-x+\frac{1}{2}, y+\frac{1}{2},-z+\frac{3}{2}$; (ix) $x-\frac{1}{2},-y+\frac{1}{2}$, $z+\frac{1}{2}$.]

triangular bipyramid $[\mathrm{Cu}-\mathrm{O}$ bond-length range of 1.9088 (9) to $2.1939(9) \AA]$ and to an $\left[\mathrm{InO}_{6}\right.$ ] octahedron [ $\mathrm{In}-\mathrm{O}$ bond lengths range from $2.1028(10)$ to $2.2051(9) \AA]$, and is completed by a distorted $\left[\mathrm{NaO}_{4}\right]$ polyhedron (Fig. 1 ). The $\mathrm{P}-$ $\mathrm{O}$ bond lengths in the two phosphate tetrahedra are similar and comparable with those of similar phosphates. However, the $\mathrm{P} 1-\mathrm{O}$ distances, varying between $1.5035(10)$ and 1.5729 (9) $\AA$, indicate a somewhat higher distortion of this tetrahedron than the P2-O distances [between 1.5297 (9) and 1.5488 (9) ^] of the other tetrahedron.

In this phosphate, two $\left[\mathrm{CuO}_{5}\right]$ triangular bipyramids share one edge to form a $\left[\mathrm{Cu}_{2} \mathrm{O}_{8}\right]$ dimer, the ends of which are linked to two $\mathrm{P} \mathrm{O}_{4}$ tetrahedra by edge-sharing. The obtained $\left[\mathrm{Cu}_{2} \mathrm{P}_{2} \mathrm{O}_{12}\right]$ groups are linked together via the vertices to form sheets extending parallel to (10) $)$, as shown in Fig. 2. On the

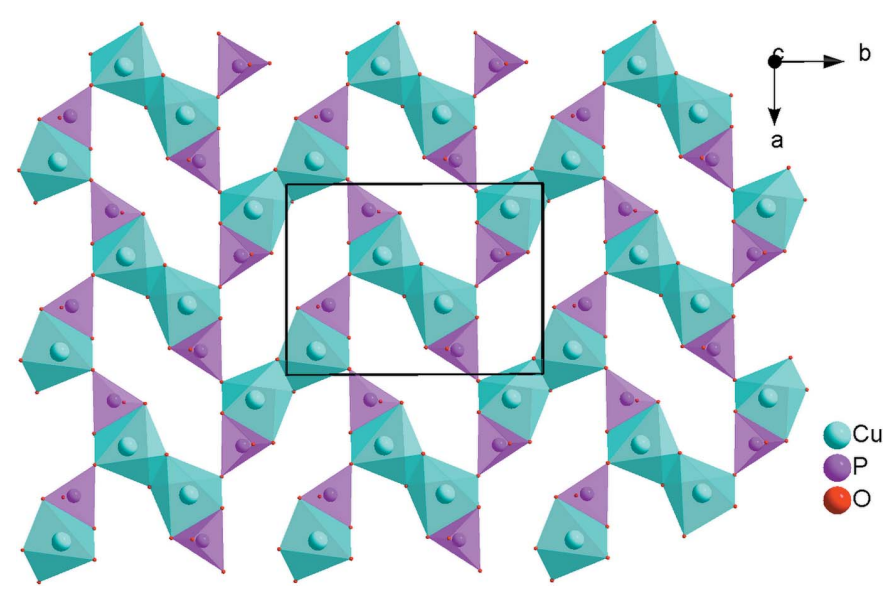

Figure 2

Projection along [001] of $\left[\mathrm{Cu}_{2} \mathrm{P}_{2} \mathrm{O}_{12}\right]$ copper phosphate sheets in the crystal structure of $\mathrm{NaCuIn}\left(\mathrm{PO}_{4}\right)_{2}$.

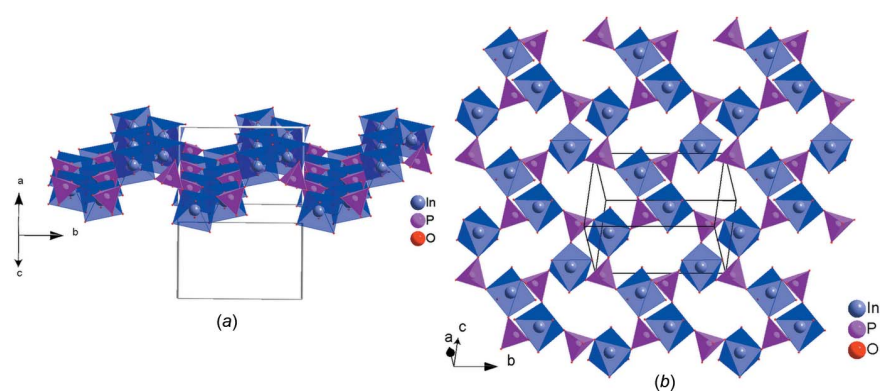

Figure 3

(a) A view approximately along [101] showing the undulating layer formed by $\left[\mathrm{InO}_{6}\right]$ octahedra linked to $\mathrm{PO}_{4}$ tetrahedra and $(b)$ a projection of this layer onto (101).

other hand, the $\left[\mathrm{InO}_{6}\right]$ octahedra and the $\mathrm{P}_{2} \mathrm{O}_{4}$ tetrahedra are interconnected through common vertices to build up an undulating layer extending in the same direction (Fig. 3). The copper phosphate layers are sandwiched between the undulating indium phosphate layers. By sharing corners and edges, an alternating stacking of the layers along [101] leads to a three-dimensional framework structure with channels in which the $\mathrm{Na}^{+}$cations are located (Fig. 4). The four nearest oxygen atoms around the alkali metal cation form a distorted disphenoid with $\mathrm{Na}-\mathrm{O}$ distances between 2.3213 (12) and 2.4275 (11) ̊ (Fig. 1).

$\mathrm{NaCuIn}\left(\mathrm{PO}_{4}\right)_{2}$ is isotypic with $\mathrm{KCuFe}\left(\mathrm{PO}_{4}\right)_{2}$ (Badri et al., 2011), whereby potassium is substituted by sodium and iron by indium. However, we note a significant difference in the coordination number of sodium and potassium in the two structures. Whereas sodium has a fourfold coordination in $\mathrm{NaCuIn}\left(\mathrm{PO}_{4}\right)_{2}$, potassium is surrounded by nine oxygen atoms in $\mathrm{KCuFe}\left(\mathrm{PO}_{4}\right)_{2}$ because of its greater ionic radius.

Bond-valence-sum calculations (Brown \& Altermatt, 1985) are in good agreement with the expected values (in valence units) for sodium(I), copper(II), indium(III) and the phosphorus(V) cations, viz. $\mathrm{Na}^{\mathrm{I}}=0.845(2), \mathrm{Cu}^{\mathrm{II}}=2.102(3)$, $\mathrm{In}^{\mathrm{III}}=3.152(4), \mathrm{P}^{\mathrm{V}}=4.930(8)$, and $\mathrm{P} 2^{\mathrm{V}}=4.992(8)$. For the

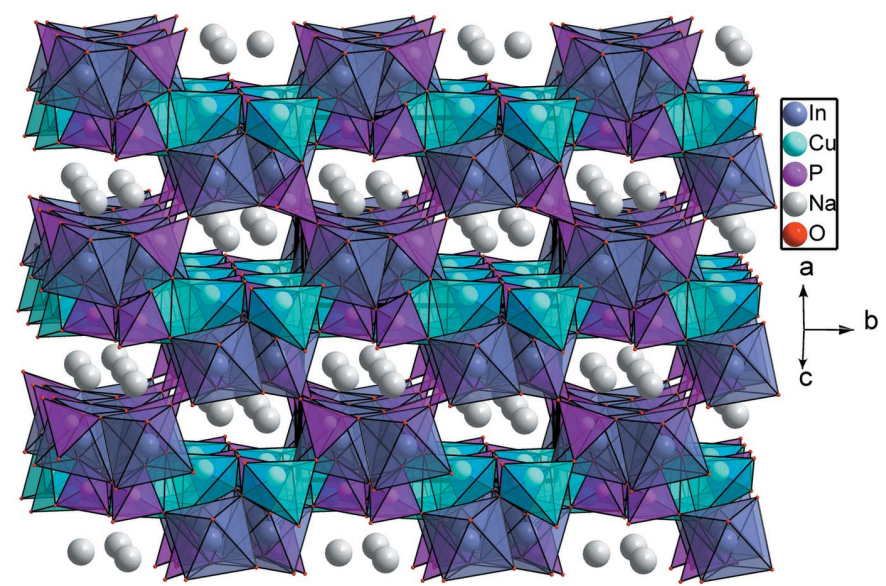

Figure 4

The sodium cations located in channels running parallel to [101] in the crystal structure of $\mathrm{NaCuIn}\left(\mathrm{PO}_{4}\right)_{2}$. 
oxygen anions, the calculated values range between 1.940 (5) and $2.076(3)$.

\section{Database survey}

Phosphate-based materials with general formula $A M^{\mathrm{II}} M^{\prime I I I}\left(\mathrm{PO}_{4}\right)_{2}$ commonly show crystal structures where channels or, more rarely, layers are formed by the $\left[M^{\mathrm{II}} M^{\prime \mathrm{II}}\left(\mathrm{PO}_{4}\right)_{2}\right]^{-}$framework to delimit suitable environments to accommodate the $A^{+}$cations. A recent survey given by Yakubovich et al. (2019) revealed that all compounds of the morphotropic series $A M^{\mathrm{II}} M^{\prime \mathrm{III}}\left(\mathrm{PO}_{4}\right)_{2}$, where $A=\mathrm{Na}, \mathrm{K}, \mathrm{Rb}$ or $\mathrm{NH}_{4}, M^{\prime \prime}=\mathrm{Cu}, \mathrm{Ni}, \mathrm{Co}, \mathrm{Fe}, \mathrm{Zn}$ or $\mathrm{Mg}$ and $M^{\prime \prime \prime}=\mathrm{Fe}, \mathrm{Al}$ or $\mathrm{Ga}$, crystallize in the monoclinic crystal system and can be classified into seven subgroups according to their structure types, viz. (i) $\mathrm{KNiFe}\left(\mathrm{PO}_{4}\right)_{2}$ (space-group type $P 2_{1} / c, Z=4$; Strutynska et al., 2014); (ii) $\mathrm{KFe}^{\mathrm{II}} \mathrm{Fe}^{\mathrm{III}}\left(\mathrm{PO}_{4}\right)_{2}$ (space-group type $P 2_{1} / c, Z=4$; Yakubovich et al., 1986); (iii) $\left(\mathrm{NH}_{4}\right) \mathrm{Fe}^{\mathrm{II}-}$ $\mathrm{Fe}^{\mathrm{III}}\left(\mathrm{PO}_{4}\right)_{2}$ (space-group type $C 2 / c, Z=16$; Boudin \& Lii, 1998); (iv) $\mathrm{K}(\mathrm{Co}, \mathrm{Al})_{2}\left(\mathrm{PO}_{4}\right)_{2}$ (space-group type $C 2 / c, Z=8$; Chen et al., 1997); (v) $\left(\mathrm{NH}_{4}\right)(\mathrm{Zn}, \mathrm{Ga})_{2}\left(\mathrm{PO}_{4}\right)_{2}$ (space-group type $P 2_{1} / a, Z=4$; Logar et al., 2001); (vi) $\mathrm{KMgFe}\left(\mathrm{PO}_{4}\right)_{2}$ (space-group type $C 2 / c, Z=4$; Badri et al., 2009); (vii) $\mathrm{NaZnAl}\left(\mathrm{PO}_{4}\right)_{2}$ (space-group type $P 2_{1} / c, Z=4$; Yakubovich et al., 2019). $\mathrm{NaCuIn}\left(\mathrm{PO}_{4}\right)_{2}$ belongs to the second subgroup of this classification.

In addition, the structures of certain members of this phosphate family are similar to those of the zeolite- $A B \mathrm{~W}$ structural type (Badri et al., 2014). When the trivalent cation is lanthanum or yttrium, the crystal structures $\mathrm{K} M^{\mathrm{II}} \mathrm{La}\left(\mathrm{PO}_{4}\right)_{2}$ $\left(M^{\mathrm{II}}=\mathrm{Mg}\right.$ or $\left.\mathrm{Zn}\right)$ are isotypes of the monazite monoclinic structure of $\mathrm{LaPO}_{4}$ with space-group type $P 2_{1} / n$ (Pan et al., 2006; Tie et al., 1995), while $\mathrm{KMgY}\left(\mathrm{PO}_{4}\right)_{2}$ turns out to be an isotype of the xenotime structure $\mathrm{YPO}_{4}$ adopting a tetragonal symmetry with space-group type $I 4_{1} /$ amd (Tie et al., 1996).

\section{Synthesis and crystallization}

Stoichiometric amounts of $\mathrm{NaNO}_{3}, \mathrm{CuO}, \mathrm{In}_{2} \mathrm{O}_{3}$ and $\mathrm{NH}_{4} \mathrm{H}_{2} \mathrm{PO}_{4}$ as precursors in the molar ratio 1:1:0.5:2 were ground in an agate mortar and pre-heated at 473 and $673 \mathrm{~K}$ in a platinum crucible to eliminate gaseous products. The resulting powder was subsequently heated to a temperature of $1473 \mathrm{~K}$. The product was then cooled to room temperature at a rate of $5 \mathrm{~K} \mathrm{~h}^{-1}$. The obtained product contained green single crystals corresponding to the title phosphate.

\section{Refinement}

Crystal data, data collection and structure refinement details are summarized in Table 1.

Labelling of atoms and their coordinates were adapted from isotypic $\mathrm{KCuFe}\left(\mathrm{PO}_{4}\right)_{2}$ (Badri et al., 2011). Since not all atoms in the latter description are part of one unit cell, a translation by $(z+1)$ relative to the original coordinates brings all corresponding atoms inside one unit cell. Moreover, oxygen
Table 1

Experimental details.

\begin{tabular}{|c|c|}
\hline \multicolumn{2}{|l|}{ Crystal data } \\
\hline Chemical formula & $\mathrm{NaCuIn}\left(\mathrm{PO}_{4}\right)_{2}$ \\
\hline$M_{\mathrm{r}}$ & 391.29 \\
\hline Crystal system, space group & Monoclinic, $P 2_{1} / n$ \\
\hline Temperature $(\mathrm{K})$ & 296 \\
\hline$a, b, c(\AA)$ & $8.2563(3), 10.1382(4), 8.8060(3)$ \\
\hline$\beta\left(^{\circ}\right)$ & $114.444(1)$ \\
\hline$V\left(\AA^{3}\right)$ & $671.03(4)$ \\
\hline$Z$ & 4 \\
\hline Radiation type & Мo $K \alpha$ \\
\hline$\mu\left(\mathrm{mm}^{-1}\right)$ & 7.16 \\
\hline Crystal size $(\mathrm{mm})$ & $0.34 \times 0.25 \times 0.19$ \\
\hline \multicolumn{2}{|l|}{ Data collection } \\
\hline Diffractometer & Bruker X8 APEX Diffractometer \\
\hline Absorption correction & $\begin{array}{l}\text { Multi-scan (SADABS; Krause et } \\
\quad \text { al., 2015) }\end{array}$ \\
\hline$T_{\min }, T_{\max }$ & $0.528,0.747$ \\
\hline $\begin{array}{l}\text { No. of measured, independent and } \\
\text { observed }[I>2 \sigma(I)] \text { reflections }\end{array}$ & 24292, 3106, 2996 \\
\hline$R_{\text {int }}$ & 0.026 \\
\hline$(\sin \theta / \lambda)_{\max }\left(\AA^{-1}\right)$ & 0.820 \\
\hline \multicolumn{2}{|l|}{ Refinement } \\
\hline$R\left[F^{2}>2 \sigma\left(F^{2}\right)\right], w R\left(F^{2}\right), S$ & $0.013,0.033,1.11$ \\
\hline No. of reflections & 3106 \\
\hline No. of parameters & 119 \\
\hline$\Delta \rho_{\max }, \Delta \rho_{\min }\left(\mathrm{e} \AA^{-3}\right)$ & $0.66,-0.59$ \\
\hline
\end{tabular}

Computer programs: APEX2 and SAINT (Bruker, 2009), SHELXT2014/5 (Sheldrick, 2015a), SHELXL2018/3 (Sheldrick, 2015b), ORTEP-3 for Windows (Farrugia, 2012), DIAMOND (Brandenburg, 2006) and publCIF (Westrip, 2010).

atoms $\mathrm{O} 11$ and $\mathrm{O} 14$ were translated by $\left(x-\frac{1}{2},-y+\frac{1}{2}, z-\frac{1}{2}\right)$ and ( $x, y, z-1)$, respectively, to be linked directly to $\mathrm{P} 1$.

The maximum and minimum electron densities in the final difference-Fourier map are at $0.70 \AA$ from $\mathrm{O} 14$ and $0.50 \AA$ from $\mathrm{Cu} 1$, respectively.

\section{Acknowledgements}

The authors thank the Unit of Support for Technical and Scientific Research (UATRS, CNRST) for the X-ray measurements.

\section{Funding information}

Mohammed V University, Rabat, Morocco, is thanked for financial support.

\section{References}

Alhakmi, G., Assani, A., Saadi, M. \& El Ammari, L. (2015). Acta Cryst. E71, 154-156.

Assani, A., Saadi, M., Zriouil, M. \& El Ammari, L. (2010). Acta Cryst. E66, i86-i87.

Badri, A., Hidouri, M., López, M. L., Pico, C., Wattiaux, A. \& Amara, M. B. (2011). J. Solid State Chem. 184, 937-944.

Badri, A., Hidouri, M., López, M. L., Veiga, M. L., Wattiaux, A. \& Amara, M. B. (2009). Solid State Ionics, 180, 1558-1563.

Badri, A., Hidouri, M., Wattiaux, A., López, M. L., Veiga, M. L. \& Amara, M. B. (2014). Mater. Res. Bull. 55, 61-66.

Boudin, S. \& Lii, K.-H. (1998). Inorg. Chem. 37, 799-803.

Brandenburg, K. (2006). DIAMOND. Crystal Impact GbR, Bonn, Germany.

Brown, I. D. \& Altermatt, D. (1985). Acta Cryst. B41, 244-247. 
Bruker (2009). APEX2 and SAINT. Bruker AXS Inc., Madison, Wisconsin, USA.

Cheetham, A. K., Férey, G. \& Loiseau, T. (1999). Angew. Chem. Int. Ed. 38, 3268-3292.

Chen, X.-A., Zhao, L., Li, Y., Guo, F. \& Chen, B.-M. (1997). Acta Cryst. C53, 1754-1756.

Farrugia, L. J. (2012). J. Appl. Cryst. 45, 849-854.

Han, M. H., Gonzalo, E., Singh, G. \& Rojo, T. (2015). Energy Environ. Sci. 8, 81-102.

Khmiyas, J., Assani, A., Saadi, M. \& El Ammari, L. (2015). Acta Cryst. E71, 55-57.

Krause, L., Herbst-Irmer, R., Sheldrick, G. M. \& Stalke, D. (2015). J. Appl. Cryst. 48, 3-10.

Logar, N. Z., Mrak, M., Kaučič, V. \& Golobič, A. (2001). J. Solid State Chem. 156, 480-486.

Manos, M. J., Iyer, R. G., Quarez, E., Liao, J. H. \& Kanatzidis, M. G. (2005). Angew. Chem. Int. Ed. 44, 3552-3555.

Manos, M. J., Malliakas, C. D. \& Kanatzidis, M. G. (2007). Chem. Eur. J. 13, 51-58.
Pan, Y., Zhang, Q. \& Jiang, Z. (2006). Mater. Sci. Eng. B, 133, 186190.

Plabst, M., McCusker, L. B. \& Bein, T. (2009). J. Am. Chem. Soc. 131, 18112-18118.

Sheldrick, G. M. (2015a). Acta Cryst. C71, 3-8.

Sheldrick, G. M. (2015b). Acta Cryst. A71, 3-8.

Stadie, N. P., Wang, S., Kravchyk, K. V. \& Kovalenko, M. V. (2017). ACS Nano, 11, 1911-1919.

Strutynska, N. Yu., Zatovsky, I. V., Baumer, V. N., Ogorodnyk, I. V. \& Slobodyanik, N. S. (2014). Acta Cryst. C70, 160-164.

Tie, S., Su, Q. \& Yu, Y. (1995). Phys. Status Solidi A, 147, 267-276.

Tie, S., Su, Q., Yu, Y. \& Ma, J. (1996). Chin. J. Chem. 14, 25-30.

Westrip, S. P. (2010). J. Appl. Cryst. 43, 920-925.

Yakubovich, O., Kiriukhina, G., Volkov, A. \& Dimitrova, O. (2019). Acta Cryst. C75, 514-522.

Yakubovich, O. V., Evdokimova, O. A., Mel'nikov, O. K. \& Simonov, M. A. (1986). Kristallografiya, 31, 906-912.

Yang, Z., Bai, Q., Li, T., Xu, S., Dong, H., Wang, Z. \& Li, P. (2016). Optik, 127, 9338-9343. 


\section{supporting information}

Acta Cryst. (2020). E76, 366-369 [https://doi.org/10.1107/S2056989020001929]

Synthesis and crystal structure of $\mathrm{NaCuln}\left(\mathrm{PO}_{4}\right)_{2}$

Elhassan Benhsina, Jamal Khmiyas, Said Ouaatta, Abderrazzak Assani, Mohamed Saadi and

\section{Lahcen El Ammari}

Computing details

Data collection: APEX2 (Bruker, 2009); cell refinement: SAINT (Bruker, 2009); data reduction: SAINT (Bruker, 2009); program(s) used to solve structure: SHELXT2014/5 (Sheldrick, 2015a); program(s) used to refine structure:

SHELXL2018/3 (Sheldrick, 2015b); molecular graphics: ORTEP-3 for Windows (Farrugia, 2012), DIAMOND

(Brandenburg, 2006); software used to prepare material for publication: publCIF (Westrip, 2010).

Sodium copper(II) indium bis[phosphate(V)]

Crystal data

$\mathrm{NaCuIn}\left(\mathrm{PO}_{4}\right)_{2}$

$M_{r}=391.29$

Monoclinic, $P 2_{1} / n$

$a=8.2563(3) \AA$

$b=10.1382(4) \AA$

$c=8.8060(3) \AA$

$\beta=114.444(1)^{\circ}$

$V=671.03(4) \AA^{3}$

$Z=4$

$F(000)=732$

$D_{\mathrm{x}}=3.873 \mathrm{Mg} \mathrm{m}^{-3}$

Mo $K \alpha$ radiation, $\lambda=0.71073 \AA$

Cell parameters from 3106 reflections

$\theta=2.9-35.6^{\circ}$

$\mu=7.16 \mathrm{~mm}^{-1}$

$T=296 \mathrm{~K}$

Block, green

$0.34 \times 0.25 \times 0.19 \mathrm{~mm}$

\section{Data collection}

Bruker X8 APEX Diffractometer

Radiation source: fine-focus sealed tube

Graphite monochromator

$\varphi$ and $\omega$ scans

Absorption correction: multi-scan

(SADABS; Krause et al., 2015)

$T_{\min }=0.528, T_{\max }=0.747$

24292 measured reflections

\section{Refinement}

Refinement on $F^{2}$

Least-squares matrix: full

$R\left[F^{2}>2 \sigma\left(F^{2}\right)\right]=0.013$

$w R\left(F^{2}\right)=0.033$

$S=1.11$

3106 reflections

119 parameters

0 restraints
3106 independent reflections

2996 reflections with $I>2 \sigma(I)$

$R_{\text {int }}=0.026$

$\theta_{\text {max }}=35.6^{\circ}, \theta_{\min }=2.9^{\circ}$

$h=-12 \rightarrow 13$

$k=-16 \rightarrow 16$

$l=-14 \rightarrow 14$ $w=1 /\left[\sigma^{2}\left(F_{\mathrm{o}}{ }^{2}\right)+(0.0139 P)^{2}+0.5758 P\right]$
$\quad$ where $P=\left(F_{\mathrm{o}}^{2}+2 F_{\mathrm{c}}{ }^{2}\right) / 3$
$(\Delta / \sigma)_{\max }=0.004$
$\Delta \rho_{\max }=0.66 \mathrm{e} \AA^{-3}$
$\Delta \rho_{\min }=-0.59 \mathrm{e} \AA^{-3}$
Extinction correction: SHELXL-2018/3
$\quad(\operatorname{Sheldrick}, 2015 \mathrm{~b})$,
$\quad \mathrm{Fc}^{*}=\mathrm{kFc}\left[1+0.001 \times \mathrm{xc}^{2} \lambda^{3} / \mathrm{sin}(2 \theta)\right]^{-1 / 4}$
Extinction coefficient: $0.0093(3)$ 


\section{Special details}

Geometry. All esds (except the esd in the dihedral angle between two 1.s. planes) are estimated using the full covariance matrix. The cell esds are taken into account individually in the estimation of esds in distances, angles and torsion angles; correlations between esds in cell parameters are only used when they are defined by crystal symmetry. An approximate (isotropic) treatment of cell esds is used for estimating esds involving l.s. planes.

Fractional atomic coordinates and isotropic or equivalent isotropic displacement parameters $\left(\AA^{2}\right)$

\begin{tabular}{lllll}
\hline & $x$ & $y$ & $z$ & $U_{\text {iso }} * U_{\text {eq }}$ \\
\hline Na1 & $0.51418(10)$ & $-0.16856(7)$ & $1.09748(8)$ & $0.01965(13)$ \\
Cu1 & $0.37225(2)$ & $0.11940(2)$ & $0.45881(2)$ & $0.00706(3)$ \\
In1 & $0.00214(2)$ & $0.12812(2)$ & $0.73403(2)$ & $0.00463(3)$ \\
P1 & $0.12997(4)$ & $0.17027(3)$ & $0.15664(4)$ & $0.00494(5)$ \\
O11 & $-0.03216(13)$ & $0.25215(10)$ & $0.13588(12)$ & $0.01037(15)$ \\
O12 & $0.30223(12)$ & $0.24790(9)$ & $0.27141(11)$ & $0.00812(14)$ \\
O13 & $0.14612(12)$ & $0.04925(9)$ & $0.27222(11)$ & $0.00824(14)$ \\
O14 & $0.13759(14)$ & $0.12965(10)$ & $-0.00448(12)$ & $0.01159(16)$ \\
P2 & $0.28460(4)$ & $-0.08358(3)$ & $0.66933(4)$ & $0.00448(5)$ \\
O21 & $0.11495(13)$ & $-0.13653(9)$ & $0.52826(12)$ & $0.00993(15)$ \\
O22 & $0.37706(12)$ & $-0.19241(8)$ & $0.79630(11)$ & $0.00787(14)$ \\
O23 & $0.24068(13)$ & $0.03111(9)$ & $0.75876(12)$ & $0.00903(15)$ \\
O24 & $0.41607(13)$ & $-0.03247(9)$ & $0.59836(12)$ & $0.00962(15)$ \\
\end{tabular}

Atomic displacement parameters $\left(\AA^{2}\right)$

\begin{tabular}{lllllll}
\hline & $U^{11}$ & $U^{22}$ & $U^{33}$ & $U^{12}$ & $U^{13}$ & $U^{23}$ \\
\hline $\mathrm{Na} 1$ & $0.0288(3)$ & $0.0145(3)$ & $0.0146(3)$ & $-0.0042(2)$ & $0.0079(3)$ & $-0.0035(2)$ \\
$\mathrm{Cu} 1$ & $0.00887(7)$ & $0.00557(6)$ & $0.00492(6)$ & $-0.00104(4)$ & $0.00104(5)$ & $0.00126(4)$ \\
$\mathrm{In} 1$ & $0.00517(4)$ & $0.00405(4)$ & $0.00432(4)$ & $-0.00022(2)$ & $0.00161(3)$ & $-0.00042(2)$ \\
$\mathrm{P} 1$ & $0.00552(11)$ & $0.00490(11)$ & $0.00367(11)$ & $-0.00020(9)$ & $0.00119(9)$ & $0.00053(8)$ \\
$\mathrm{O} 11$ & $0.0096(4)$ & $0.0125(4)$ & $0.0094(4)$ & $0.0054(3)$ & $0.0044(3)$ & $0.0042(3)$ \\
$\mathrm{O} 12$ & $0.0085(4)$ & $0.0077(3)$ & $0.0063(3)$ & $-0.0034(3)$ & $0.0012(3)$ & $0.0008(3)$ \\
$\mathrm{O} 13$ & $0.0091(4)$ & $0.0055(3)$ & $0.0071(3)$ & $-0.0028(3)$ & $0.0004(3)$ & $0.0020(3)$ \\
$\mathrm{O} 14$ & $0.0129(4)$ & $0.0165(4)$ & $0.0043(3)$ & $0.0025(3)$ & $0.0025(3)$ & $-0.0014(3)$ \\
$\mathrm{P} 2$ & $0.00536(11)$ & $0.00392(11)$ & $0.00410(11)$ & $0.00089(8)$ & $0.00189(9)$ & $0.00025(8)$ \\
$\mathrm{O} 21$ & $0.0096(4)$ & $0.0119(4)$ & $0.0054(3)$ & $-0.0026(3)$ & $0.0002(3)$ & $-0.0013(3)$ \\
$\mathrm{O} 22$ & $0.0109(4)$ & $0.0058(3)$ & $0.0073(3)$ & $0.0032(3)$ & $0.0041(3)$ & $0.0027(3)$ \\
$\mathrm{O} 23$ & $0.0088(4)$ & $0.0071(3)$ & $0.0113(4)$ & $0.0018(3)$ & $0.0042(3)$ & $-0.0034(3)$ \\
$\mathrm{O} 24$ & $0.0101(4)$ & $0.0092(4)$ & $0.0125(4)$ & $0.0030(3)$ & $0.0077(3)$ & $0.0056(3)$ \\
& & & & & & \\
\hline
\end{tabular}

Geometric parameters $\left(\stackrel{\AA}{\circ}{ }^{\circ}\right)$

\begin{tabular}{llll}
\hline $\mathrm{Na} 1-\mathrm{O} 21^{\mathrm{i}}$ & $2.3213(12)$ & $\mathrm{In} 1-\mathrm{O} 22^{\text {viii }}$ & $2.1441(9)$ \\
$\mathrm{Na} 1-\mathrm{O} 23^{\text {ii }}$ & $2.3496(12)$ & $\mathrm{I} 1-\mathrm{O} 13^{\text {vii }}$ & $2.1632(9)$ \\
$\mathrm{Na} 1-\mathrm{O} 22$ & $2.4268(11)$ & $\mathrm{I} 1-\mathrm{O} 12^{\text {ix }}$ & $2.2051(9)$ \\
$\mathrm{Na} 1-\mathrm{O} 11^{\text {iii }}$ & $2.4275(11)$ & $\mathrm{P} 1-\mathrm{O} 14$ & $1.5035(10)$ \\
$\mathrm{Cu} 1-\mathrm{O} 24$ & $1.9088(9)$ & $\mathrm{P} 1-\mathrm{O} 11$ & $1.5205(10)$ \\
$\mathrm{Cu} 1-\mathrm{O} 11^{\text {iv }}$ & $1.9317(9)$ & $\mathrm{P} 1-\mathrm{O} 13$ & $1.5642(9)$
\end{tabular}




\begin{tabular}{|c|c|c|c|}
\hline $\mathrm{Cu} 1-\mathrm{O} 12$ & $1.9913(9)$ & $\mathrm{P} 1-\mathrm{O} 12$ & $1.5729(9)$ \\
\hline $\mathrm{Cu} 1-\mathrm{O} 13$ & $2.0378(9)$ & $\mathrm{P} 2-\mathrm{O} 23$ & $1.5297(9)$ \\
\hline $\mathrm{Cu} 1-\mathrm{O} 24^{\mathrm{v}}$ & $2.1939(9)$ & $\mathrm{P} 2-\mathrm{O} 22$ & $1.5310(9)$ \\
\hline $\mathrm{In} 1-\mathrm{O} 14^{\mathrm{vi}}$ & $2.1028(10)$ & $\mathrm{P} 2-\mathrm{O} 21$ & $1.5340(10)$ \\
\hline $\mathrm{In} 1-\mathrm{O} 21^{\mathrm{vii}}$ & $2.1044(9)$ & $\mathrm{P} 2-\mathrm{O} 24$ & $1.5488(9)$ \\
\hline $\mathrm{In} 1-\mathrm{O} 23$ & $2.1303(9)$ & & \\
\hline $\mathrm{O} 21^{\mathrm{i}}-\mathrm{Na} 1-\mathrm{O} 23^{\mathrm{ii}}$ & $108.90(4)$ & $\mathrm{O} 14^{\mathrm{vi}}-\mathrm{In} 1-\mathrm{O} 13^{\mathrm{vii}}$ & $94.18(4)$ \\
\hline $\mathrm{O} 21^{\mathrm{i}}-\mathrm{Na} 1-\mathrm{O} 22$ & $71.58(4)$ & $\mathrm{O} 21^{\mathrm{vii}-\mathrm{In} 1-\mathrm{O} 13^{\mathrm{vii}}}$ & $90.44(4)$ \\
\hline $\mathrm{O} 23^{\mathrm{ii}}-\mathrm{Na} 1-\mathrm{O} 22$ & $123.80(4)$ & $\mathrm{O} 23-\mathrm{In} 1-\mathrm{O} 13^{\mathrm{vii}}$ & $96.21(4)$ \\
\hline 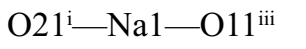 & 94.99 (4) & $\mathrm{O} 22^{\text {viii-In } 1-\mathrm{O} 13^{\mathrm{vii}}}$ & $171.80(3)$ \\
\hline $\mathrm{O} 23^{\mathrm{ii}}-\mathrm{Na} 1-\mathrm{O} 11^{\mathrm{iii}}$ & $88.91(4)$ & $\mathrm{O} 14^{\mathrm{vi}}-\mathrm{In} 1-\mathrm{O} 12^{\mathrm{ix}}$ & $85.65(4)$ \\
\hline $\mathrm{O} 22-\mathrm{Na} 1-\mathrm{O} 11^{\mathrm{iii}}$ & $146.95(4)$ & $\mathrm{O} 21^{\mathrm{vii}-\mathrm{In} 1-\mathrm{O} 12^{\mathrm{ix}}}$ & $96.23(4)$ \\
\hline $\mathrm{O} 24-\mathrm{Cu} 1-\mathrm{O} 11^{\mathrm{iv}}$ & $96.82(4)$ & $\mathrm{O} 23-\mathrm{In} 1-\mathrm{O} 12^{\mathrm{ix}}$ & $164.87(3)$ \\
\hline $\mathrm{O} 24-\mathrm{Cu} 1-\mathrm{O} 12$ & $166.88(4)$ & $\mathrm{O} 22^{\mathrm{vii}-\mathrm{In} 1-\mathrm{O} 12^{\mathrm{ix}}}$ & $87.16(3)$ \\
\hline $\mathrm{O} 11^{\mathrm{iv}}-\mathrm{Cu} 1-\mathrm{O} 12$ & $96.28(4)$ & 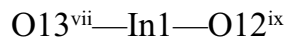 & $91.55(3)$ \\
\hline $\mathrm{O} 24-\mathrm{Cu} 1-\mathrm{O} 13$ & $95.96(4)$ & $\mathrm{O} 14-\mathrm{P} 1-\mathrm{O} 11$ & $114.50(6)$ \\
\hline $\mathrm{O} 11^{\mathrm{iv}}-\mathrm{Cu} 1-\mathrm{O} 13$ & $144.90(4)$ & $\mathrm{O} 14-\mathrm{P} 1-\mathrm{O} 13$ & $111.94(5)$ \\
\hline $\mathrm{O} 12-\mathrm{Cu} 1-\mathrm{O} 13$ & $72.85(3)$ & $\mathrm{O} 11-\mathrm{P} 1-\mathrm{O} 13$ & $109.89(5)$ \\
\hline $\mathrm{O} 24-\mathrm{Cu} 1-\mathrm{O} 24^{\mathrm{v}}$ & $82.35(4)$ & $\mathrm{O} 14-\mathrm{P} 1-\mathrm{O} 12$ & $111.32(5)$ \\
\hline $\mathrm{O} 11^{\mathrm{iv}}-\mathrm{Cu} 1-\mathrm{O} 24^{\mathrm{v}}$ & $110.97(4)$ & $\mathrm{O} 11-\mathrm{P} 1-\mathrm{O} 12$ & $108.72(5)$ \\
\hline $\mathrm{O} 12-\mathrm{Cu} 1-\mathrm{O} 24^{\mathrm{v}}$ & $93.34(4)$ & $\mathrm{O} 13-\mathrm{P} 1-\mathrm{O} 12$ & $99.40(5)$ \\
\hline $\mathrm{O} 13-\mathrm{Cu} 1-\mathrm{O} 24^{\mathrm{v}}$ & $103.05(4)$ & $\mathrm{O} 23-\mathrm{P} 2-\mathrm{O} 22$ & $108.94(5)$ \\
\hline $\mathrm{O} 14^{\mathrm{vi}}-\mathrm{In} 1-\mathrm{O} 21^{\mathrm{vii}}$ & $174.97(4)$ & $\mathrm{O} 23-\mathrm{P} 2-\mathrm{O} 21$ & $110.57(5)$ \\
\hline $\mathrm{O} 14^{\mathrm{vi}}-\mathrm{In} 1-\mathrm{O} 23$ & $80.87(4)$ & $\mathrm{O} 22-\mathrm{P} 2-\mathrm{O} 21$ & $110.58(5)$ \\
\hline 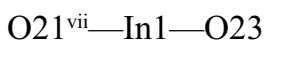 & $96.68(4)$ & $\mathrm{O} 23-\mathrm{P} 2-\mathrm{O} 24$ & $107.97(5)$ \\
\hline $\mathrm{O} 14^{\mathrm{vi} \_\mathrm{In} 1-\mathrm{O} 22^{\mathrm{viii}}}$ & $93.79(4)$ & $\mathrm{O} 22-\mathrm{P} 2-\mathrm{O} 24$ & $108.36(5)$ \\
\hline $\mathrm{O} 21^{\mathrm{vii}}-\mathrm{In} 1-\mathrm{O} 22^{\text {viii }}$ & $81.66(3)$ & $\mathrm{O} 21-\mathrm{P} 2-\mathrm{O} 24$ & $110.35(5)$ \\
\hline $\mathrm{O} 23-\mathrm{In} 1-\mathrm{O} 22^{\mathrm{viii}}$ & $86.94(4)$ & & \\
\hline
\end{tabular}

Symmetry codes: (i) $x+1 / 2,-y-1 / 2, z+1 / 2$; (ii) $-x+1,-y,-z+2$; (iii) $-x+1 / 2, y-1 / 2,-z+3 / 2$; (iv) $x+1 / 2,-y+1 / 2, z+1 / 2$; (v) $-x+1,-y,-z+1$; (vi) $x, y, z+1$; (vii) $-x,-y,-z+1$; (viii) $-x+1 / 2, y+1 / 2,-z+3 / 2$; (ix) $x-1 / 2,-y+1 / 2, z+1 / 2$. 